\title{
On Rayleigh Wave in Two-Temperature Generalized Thermoelastic Medium without Energy Dissipation
}

\author{
Baljeet Singh $^{1}$, Kiran Bala ${ }^{2,3}$ \\ ${ }^{1}$ Department of Mathematics, Post Graduate Government College, Chandigarh, India \\ ${ }^{2}$ Department of Mathematics, Government College, Haryana, India \\ ${ }^{3}$ Research Scholar in Mathematics, Singhania University, Rajasthan, India \\ Email: bsinghgc11@gmail.com, kirandulat@gmail.com
}

Received October 8, 2012; revised November 8, 2012; accepted November 15, 2012

\begin{abstract}
In this paper, Rayleigh surface wave is studied at a stress free thermally insulated surface of a two-temperature thermoelastic solid half-space in absence of energy dissipation. The governing equations of two-temperature generalized thermoelastic medium without energy dissipation are solved for surface wave solutions. The appropriate particular solutions are applied to the required boundary conditions to obtain the frequency equation of the Rayleigh wave. Some special cases are also derived. The non-dimensional speed is computed numerically and shown graphically to show the dependence on the frequency and two-temperature parameter.
\end{abstract}

Keywords: Two-Temperature; Generalized Thermoelasticity; Rayleigh Wave; Energy Dissipation

\section{Introduction}

Lord and Shulman [1] and Green and Lindsay [2] extended the classical dynamical coupled theory of thermoelasticity to generalized thermoelasticity theories. These theories treat heat propagation as a wave phenomenon rather than a diffusion phenomenon and predict a finite speed of heat propagation. Ignaczak and OstojaStarzewski [3] explained in detail, the above theories in their book on "Thermoelasticity with Finite Wave Speeds". The theory of thermoelasticity without energy dissipation is another generalized theory, which was formulated by Green and Naghdi [4]. It includes the isothermal displacement gradients among its independent constitutive variables and differs from the previous theories in that it does not accommodate dissipation of thermal energy. The representative theories in the range of generalized thermoelasticity are reviewed by Hetnarski and Ignaczak [5]. Wave propagation in thermoelasticity has many applications in various engineering fields. Some problems on wave propagation in coupled or generalized thermoelasticity are studied by various researchers, for example, Deresiewicz [6], Sinha and Sinha [7], Sinha and Elsibai [8,9], Sharma, et al. [10], Othman and Song [11], Singh $[12,13]$, and many more.

Gurtin and Williams $[14,15]$ suggested the second law of thermodynamics for continuous bodies in which the entropy due to heat conduction was governed by one temperature, that of the heat supply by another temperature. Based on this suggestion, Chen and Gurtin [16] and
Chen et al. $[17,18]$ formulated a theory of thermoelasticity which depends on two distinct temperatures, the conductive temperature $\Phi$ and the thermodynamic temperature $T$. The two-temperature theory involves a material parameter $a^{*}>0$. The limit $a^{*} \rightarrow 0$ implies that $\Phi \rightarrow T$ and the classical theory can be recovered from two-temperature theory. The two-temperature model has been widely used to predict the electron and phonon temperature distributions in ultrashort laser processing of metals. Warren and Chen [19] stated that these two temperatures can be equal in time-dependent problems under certain conditions, whereas $\Phi$ and $T$ are generally different in particular problems involving wave propagation. Following Boley and Tolins [20], they studied the wave propagation in the two-temperature theory of coupled thermoelasticity. They showed that the two temperatures $T$ and $\Phi$, and the strain are represented in the form of a travelling wave plus a response, which occurs instantaneously throughout the body. Puri and Jordan [21] discussed the propagation of harmonic plane waves in two temperature theory. Quintanilla and Jordan [22] presented exact solutions of two initial-boundary value problems in the two temperature theory with dual-phase-lag delay. Youssef [23] formulated a theory of two-temperature generalized thermoelasticity. Kumar and Mukhopadhyay [24] extended the work of Puri and Jordan [21] in the context of the linear theory of two-temperature generalized thermoelasticity formulated by Youssef [23]. Magana and Quintanilla [25] studied the uniqueness and growth of solutions in two-temperature generalized ther- 
moelastic theories. Recently, Youssef [26] presented a theory of two-temperature thermoelasticity without energy dissipation.

In the present paper, Youssef [26] theory is applied to study the Rayleigh wave at the thermally insulated stressfree surface of an isotropic two-temperature thermoelastic solid half-space without energy dissipation. The frequency equation of the Rayleigh wave is obtained. The frequency equation is also approximated by assuming small thermal coupling. The dependence of numerical values of non-dimensional speed of the Rayleigh wave on material parameters, frequency and two-temperature parameters is shown graphically for a particular material of the model.

\section{Basic Equations}

We consider a two-temperature thermoelastic solid halfspace in absence of energy dissipation. Following Youssef [26], the governing equations for a two-temperature generalized thermoelastic half-space without energy dissipation are

i) The heat conduction equation

$$
K^{*} \Phi_{, i i}=\rho c_{E} \ddot{\theta}+\gamma T_{0} \ddot{e}_{k k},
$$

ii) The displacement-strain relation

$$
e_{i j}=\frac{1}{2}\left(u_{i, j}+u_{j, i}\right),
$$

iii) The equation of motion

$$
\rho \ddot{u}_{i}=(\lambda+\mu) u_{j, i j}+\mu u_{i, j j}-\gamma \theta_{, i},
$$

iv) The constitutive equations

$$
\sigma_{i j}=2 \mu e_{i j}+\left(\lambda e_{k k}-\gamma \theta\right) \delta_{i j},
$$

where $\gamma=(3 \lambda+2 \mu) \alpha_{t}$, is the coupling parameter and $\alpha_{t}$, is the thermal expansion coefficient. $\lambda$ and $\mu$ are called Lame's elastic constants. $\delta_{i j}$ is the Kronecker delta. $K^{*}$ is material characteristic constant. $T$ is the mechanical temperature, $\Phi_{0}=T_{0}$ is the reference temperature. $\theta=T-T_{0}$ with $\left|\theta / T_{0}\right| \ll 1 . \sigma_{i j}$ is the stress tensor. $e_{i j}$ is the strain tensor. $\rho$ is the mass density. $c_{E}$ is the specific heat at constant strain. $u_{i}$ are the components of the displacement vector. $\Phi$ is the conductive temperature and satisfies the relation

$$
\Phi-\theta=a^{*} \Phi_{, i i},
$$

where $a^{*}>0$ is the two-temperature parameter. The superposed dots in the above equations denote the time derivatives. The subscripts followed by comma in these equations denote the space derivatives.

\section{Analytical 2D Solution}

We consider a homogeneous and isotropic two-temperature thermoelastic medium without energy dissipation of an infinite extent with Cartesian coordinates system $(x, y, z)$, which is previously at uniform temperature $T_{0}$. The origin is taken on the plane surface $z=0$ and the $z$-axis is taken normally into the medium $z \geq 0$. The surface $z=0$ is assumed stress-free and thermally insulated. The present study is restricted to the plane strain parallel to $x-z$ plane, with the displacement vector $u=\left(u_{1}, 0, u_{3}\right)$. Now, Equation (3) has the following two components in $x-z$ plane

$$
\begin{aligned}
& (\lambda+2 \mu) u_{1,11}+(\lambda+\mu) u_{3,13}+\mu u_{1,33}-\gamma \theta_{, 1}=\rho \ddot{u}_{1}, \\
& (\lambda+2 \mu) u_{3,33}+(\lambda+\mu) u_{1,13}+\mu u_{3,11}-\gamma \theta_{, 3}=\rho \ddot{u}_{3},
\end{aligned}
$$

The heat conduction Equation (1) is written in $x-z$ plane as

$$
K^{*}\left(\Phi_{, 11}+\Phi_{, 33}\right)=\rho c_{E} \frac{\partial^{2} \theta}{\partial t^{2}}+\gamma T_{0} \frac{\partial^{2}}{\partial t^{2}}\left(u_{1,1}+u_{3,3}\right),
$$

and, Equation (5) becomes,

$$
\Phi-\theta=a^{*}\left(\Phi_{, 11}+\Phi_{, 33}\right) .
$$

The displacement components $u_{1}$ and $u_{3}$ are written in terms of scalar potentials $q$ and $\psi$ as

$$
u_{1}=\frac{\partial q}{\partial x}-\frac{\partial \psi}{\partial z}, u_{3}=\frac{\partial q}{\partial z}+\frac{\partial \psi}{\partial x} .
$$

Using Equations (9) and (10) in Equations (6) to (8), we obtain

$$
\begin{gathered}
\frac{\partial^{2} q}{\partial t^{2}}=c_{1}^{2}\left(\frac{\partial^{2} q}{\partial x^{2}}+\frac{\partial^{2} q}{\partial z^{2}}\right) \\
-\frac{\gamma}{\rho}\left[\Phi-a^{*}\left(\frac{\partial^{2} \Phi}{\partial x^{2}}+\frac{\partial^{2} \Phi}{\partial z^{2}}\right)\right], \\
\frac{\partial^{2} \psi}{\partial t^{2}}=c_{2}^{2}\left(\frac{\partial^{2} \psi}{\partial x^{2}}+\frac{\partial^{2} \psi}{\partial z^{2}}\right) \\
K^{*}\left(\frac{\partial^{2} \Phi}{\partial x^{2}}+\frac{\partial^{2} \Phi}{\partial z^{2}}\right) \\
=\rho c_{E} \frac{\partial^{2} \Phi}{\partial t^{2}}-a^{*} \rho c_{E} \frac{\partial^{2}}{\partial t^{2}}\left(\frac{\partial^{2} \Phi}{\partial x^{2}}+\frac{\partial^{2} \Phi}{\partial z^{2}}\right) \\
+\gamma T_{0} \frac{\partial^{2}}{\partial t^{2}}\left(\frac{\partial^{2} q}{\partial x^{2}}+\frac{\partial^{2} q}{\partial z^{2}}\right),
\end{gathered}
$$

where $c_{1}^{2}=\frac{(\lambda+2 \mu)}{\rho}, \quad c_{2}^{2}=\frac{\mu}{\rho}$.

Using the following quantities

$$
\begin{gathered}
x^{\prime}=\frac{x}{\left(c_{1} / \omega^{*}\right)}, \quad z^{\prime}=\frac{z}{\left(c_{1} / \omega^{*}\right)}, \quad t^{\prime}=t \omega^{*}, \\
\Phi^{\prime}=\frac{\gamma \Phi}{\rho c_{1}^{2}}, \quad q^{\prime}=\frac{q}{\left(c_{1} / \omega^{*}\right)^{2}}, \quad \psi^{\prime}=\frac{\psi}{\left(c_{1} / \omega^{*}\right)^{2}},
\end{gathered}
$$




$$
a^{\prime *}=\frac{a^{*}}{\left(c_{1} / \omega^{*}\right)^{2}}
$$

where $\omega^{*}=\rho c_{E} c_{1}^{2} / K^{*}$, in Equations (11) to (13) and suppressing the primes, we obtain the Equations (11) to (13) in dimensionless form as

$$
\begin{gathered}
\frac{\partial^{2} q}{\partial t^{2}}=\left(\frac{\partial^{2} q}{\partial x^{2}}+\frac{\partial^{2} q}{\partial z^{2}}\right)-\Phi+a^{*}\left(\frac{\partial^{2} \Phi}{\partial x^{2}}+\frac{\partial^{2} \Phi}{\partial z^{2}}\right), \\
\frac{\partial^{2} \psi}{\partial t^{2}}=\frac{1}{v^{2}}\left(\frac{\partial^{2} \psi}{\partial x^{2}}+\frac{\partial^{2} \psi}{\partial z^{2}}\right), \\
\ddot{\Phi}+\varepsilon \frac{\partial^{2}}{\partial t^{2}}\left(\frac{\partial^{2} q}{\partial x^{2}}+\frac{\partial^{2} q}{\partial z^{2}}\right) \\
=\left(\frac{1}{\omega^{*}}+a^{*} \frac{\partial^{2}}{\partial t^{2}}\right)\left(\frac{\partial^{2} \Phi}{\partial x^{2}}+\frac{\partial^{2} \Phi}{\partial z^{2}}\right),
\end{gathered}
$$

where $v^{2}=\frac{c_{1}^{2}}{c_{2}^{2}}$, and

$$
\epsilon=\frac{\gamma^{2} T_{0}}{\rho^{2} c_{E} c_{1}^{2}},
$$

is the coefficient of thermoelastic coupling.

For thermoelastic surface waves in the half-space propagating in $x$-direction, the potential functions $\Phi, q$ and $\psi$ are taken in the following form

$$
(\Phi, q, \psi)=(\hat{\Phi}(z), \hat{q}(z), \hat{\psi}(z)) \exp i(\eta x-\chi t),
$$

where $\chi^{2}=\eta^{2} c^{2}, \eta$ is wave number and $c$ is the phase velocity.

Substituting Equation (18) in Equations (14) and (16), we obtain

$$
\begin{gathered}
{\left[a_{1}+\chi^{2} \epsilon D^{2}\right] \hat{q}+\left[a_{2}+\left(\frac{1}{\omega^{*}}-\chi^{2} a^{*}\right) D^{2}\right] \hat{\Phi}=0,} \\
{\left[a_{3}-D^{2}\right] \hat{q}+\left[a_{4}-a^{*} D^{2}\right] \hat{\Phi}=0,}
\end{gathered}
$$

where $D^{2}=\frac{\mathrm{d}^{2}}{\mathrm{~d} z^{2}}$, and

$$
\begin{aligned}
& a_{1}=-\chi^{2} \epsilon \eta^{2}, \quad a_{2}=\chi^{2}-\left(\frac{1}{\omega^{*}}-\chi^{2} a^{*}\right) \eta^{2}, \\
& a_{3}=\left(-\chi^{2}+\eta^{2}\right), \quad a_{4}=\left(1+a^{*} \eta^{2}\right)
\end{aligned}
$$

Eliminating $\hat{q}, \hat{\Phi}$ from Equations (19) and (20), we obtain the following auxiliary equation

$$
A_{0} D^{4}+A_{1} D^{2}+A_{2}=0 \text {, }
$$

where

$$
A_{0}=\frac{1}{\omega^{*}}-\chi^{2} a^{*}(1+\epsilon),
$$

$$
\begin{aligned}
& A_{1}=a_{2}-a_{1} a^{*}-\frac{a_{3}}{\omega^{*}}+\chi^{2}\left(a_{3} a^{*}+\epsilon a_{4}\right), \\
& A_{2}=a_{1} a_{4}-a_{2} a_{3} .
\end{aligned}
$$

With the help of Equation (21) and keeping in mind that $\hat{q}, \hat{\Phi} \rightarrow 0$ as $z \rightarrow \infty$ for surface waves, the solutions $q$, $\Phi$ are written as

$$
\begin{gathered}
q=\left[A \exp \left(-\eta \beta_{1} z\right)+B \exp \left(-\eta \beta_{2} z\right)\right] \exp \imath(\eta x-\chi t) \\
\Phi=\left[\eta_{1} A \exp \left(-\eta \beta_{1} z\right)+\eta_{2} B \exp \left(-\eta \beta_{2} z\right)\right] \exp \imath(\eta x-\chi t)
\end{gathered}
$$

where

$$
\begin{aligned}
& \beta_{1}^{2}=\frac{1}{\eta^{2}}\left[\frac{-A_{1}+\sqrt{A_{1}^{2}-4 A_{0} A_{2}}}{2 A_{0}}\right], \\
& \beta_{2}^{2}=\frac{1}{\eta^{2}}\left[\frac{-A_{1}-\sqrt{A_{1}^{2}-4 A_{0} A_{2}}}{2 A_{0}}\right],
\end{aligned}
$$

and

$$
\eta_{i}=\frac{\eta^{2}\left(1-\beta_{i}^{2}\right)-\chi^{2}}{a^{*} \eta^{2}\left(\beta_{i}^{2}-1\right)-1}, \quad(i=1,2) .
$$

Substituting Equation (18) in Equation (15) and keeping in mind that $\hat{\psi} \rightarrow 0$ as $z \rightarrow \infty$ for surface waves, we obtain the following solution

$$
\psi=C \exp \left[-\eta \beta_{3} z+\imath(\eta x-\chi t)\right]
$$

where

$$
\beta_{3}^{2}=1-c^{2} v^{2}
$$

\section{Derivation of Frequency Equation}

The mechanical and thermal conditions at the thermally insulated surface $z=0$ are

i) Vanishing of the normal stress component

$$
\sigma_{z z}=0 \text {, }
$$

ii) Vanishing of the tangential stress component

$$
\sigma_{z x}=0,
$$

iii) Vanishing of the normal heat flux component

$$
\frac{\partial \Phi}{\partial z}=0
$$

where

$$
\begin{aligned}
\sigma_{z z} & =\lambda\left(\frac{\partial^{2} q}{\partial x^{2}}+\frac{\partial^{2} q}{\partial z^{2}}\right)+2 \mu\left(\frac{\partial^{2} \psi}{\partial x \partial z}\right)+2 \mu \frac{\partial^{2} q}{\partial z^{2}} \\
& -\gamma\left[\Phi-a^{*}\left(\frac{\partial^{2} \Phi}{\partial x^{2}}+\frac{\partial^{2} \Phi}{\partial z^{2}}\right)\right],
\end{aligned}
$$




$$
\sigma_{z x}=\mu\left[2 \frac{\partial^{2} q}{\partial x \partial z}-\frac{\partial^{2} \psi}{\partial z^{2}}+\frac{\partial^{2} \psi}{\partial x^{2}}\right] .
$$

Equation (29) to (31) are written in non-dimensional form as

$$
\begin{gathered}
\lambda\left(\frac{\partial^{2} q}{\partial x^{2}}+\frac{\partial^{2} q}{\partial z^{2}}\right)+2 \mu\left(\frac{\partial^{2} \psi}{\partial x \partial z}+\frac{\partial^{2} q}{\partial z^{2}}\right) \\
-\rho c_{1}^{2}\left[\Phi-a^{*}\left(\frac{\partial^{2} \Phi}{\partial x^{2}}+\frac{\partial^{2} \Phi}{\partial z^{2}}\right)\right]=0, \\
2 \frac{\partial^{2} q}{\partial x \partial z}-\frac{\partial^{2} \psi}{\partial z^{2}}+\frac{\partial^{2} \psi}{\partial x^{2}}=0, \\
\frac{\partial \Phi}{\partial z}=0 .
\end{gathered}
$$

Making use of solutions (22), (23) and (27) for $q, \Phi, \Psi$ in the Equations (34) to (36), we obtain the following homogenous system of three equations in $A, B$ and $C$

$$
\begin{aligned}
& {\left[-\lambda \eta^{2}+\eta^{2} \beta_{1}^{2}(\lambda+2 \mu)-\rho c_{1}^{2} \eta_{1}\left\{1+a^{*} \eta^{2}\left(1-\beta_{1}^{2}\right)\right\}\right] A} \\
& +\left[-\lambda \eta^{2}+\eta^{2} \beta_{2}^{2}(\lambda+2 \mu)-\rho c_{1}^{2} \eta_{2}\left\{1+a^{*} \eta^{2}\left(1-\beta_{2}^{2}\right)\right\}\right] B \\
& -2 \imath \mu \eta^{2} \beta_{3} C=0
\end{aligned}
$$

$$
\begin{aligned}
& 2 \imath \beta_{1} A+2 \imath \beta_{2} B+\left(1+\beta_{3}^{2}\right) C=0, \\
& \eta_{1} \beta_{1} A+\eta_{2} \beta_{2} B=0,
\end{aligned}
$$

The non-trivial solution of Equations (37) to (39) exists if the determinant of the coefficients of $A, B$ and $C$ vanishes, i.e.,

$$
\begin{aligned}
& 4 \mu \eta^{2} \beta_{1} \beta_{2} \beta_{3}\left(\eta_{2}-\eta_{1}\right) \\
& -\left(1+\beta_{3}^{2}\right) \\
& \cdot\left[\eta^{2}\left\{-\lambda\left(\eta_{2} \beta_{2}-\eta_{1} \beta_{1}\right)+(\lambda+2 \mu) \beta_{1} \beta_{2}\left(\eta_{2} \beta_{1}-\eta_{1} \beta_{2}\right)\right\}\right. \\
& \left.-\rho c_{1}^{2} \eta_{1} \eta_{2}\left(\beta_{2}-\beta_{1}\right)\left\{1+a^{*} \eta^{2}\left(1+\beta_{1} \beta_{2}\right)\right\}\right]=0,
\end{aligned}
$$

which is the the frequency equation of thermoelastic Rayleigh wave in a two-temperature generalized thermoelastic medium without energy dissipation.

\section{Special Cases}

\subsection{Small Thermal Coupling}

In order to have an idea of the effect of two-temperature parameter on the speed of propagation of Rayleigh wave, we consider the case of small thermoelastic coupling. For most of materials, $\epsilon$ is small at normal temperature. Hence we can approximate the frequency equation by assuming $\epsilon \ll 1$. For $\epsilon \ll 1$, the Equations (24) and (25) are approximated as

$$
\begin{aligned}
& \beta_{1} \simeq \frac{c}{\chi} \sqrt{\frac{-b_{1}+b_{5}}{2 b_{2}}} \\
& {\left[1+\frac{\epsilon}{2}\left(\frac{\chi^{2} a^{*}}{b_{2}}+\frac{\frac{-\chi^{2} a^{*}}{c^{2}}\left(\chi^{2}+1\right)+1+\frac{1}{2} \frac{b_{4}}{b_{5}}}{-b_{1}+b_{5}}\right)\right],} \\
& \left.\beta_{2} \simeq \frac{c}{\chi} \sqrt{\frac{-\left(b_{1}+b_{5}\right)}{2 b_{2}}}\right) \\
& {\left[1+\frac{\epsilon}{2}\left(\frac{\chi^{2} a^{*}}{b_{2}}-\frac{\frac{-\chi^{2} a^{*}}{c^{2}}\left(\chi^{2}+1\right)+1+\frac{1}{2} \frac{b_{4}}{b_{5}}}{b_{1}+b_{5}}\right),\right.}
\end{aligned}
$$

where

$$
\begin{aligned}
b_{1}= & \chi^{2}+b_{2}\left(\chi^{2}-2 \eta^{2}\right), b_{2}=\frac{1}{\omega^{*}}-\chi^{2} a^{*}, \\
b_{3}= & \left(\chi^{2}-\eta^{2}\right)\left(\chi^{2}-b_{2} \eta^{2}\right) \\
b_{4}= & 2 b_{1}\left\{\eta^{2} a^{*}\left(\chi^{2}+1\right)+1\right\} \\
& +4 b_{2} b_{3} \chi^{2}\left\{\frac{a^{*}}{b_{2}}+\eta^{2}\left(\frac{1+a^{*} \eta^{2}}{b_{3}}\right)\right\}, \\
b_{5}= & \sqrt{b_{1}^{2}-4 b_{2} b_{3}} .
\end{aligned}
$$

With the help of these approximations for $\beta_{1}$ and $\beta_{2}$, the coupling coefficients $\eta_{1}$ and $\eta_{2}$ are approximated and hence the frequency Equation (40) is approximated.

\subsection{Isotropic Elastic Case}

If we neglect thermal parameters, then the frequency Equation (40) reduces to

$$
\left(2-c^{2} v^{2}\right)^{2}=4 \sqrt{1-c^{2}} \sqrt{1-c^{2} v^{2}},
$$

which is the frequency equation of Rayleigh wave for an isotropic elastic case.

\section{Numerical Example}

If we put $c^{2}=c^{* 2}+\epsilon\left(\xi_{1}+i \xi_{2}\right)$, where $c^{*}$ is the classical Rayleigh wave velocity and $\xi_{1}$ and $\xi_{2}$ are two reals, then

$$
\eta=\frac{\chi}{c^{*}}\left(1-\frac{\epsilon \xi_{1}}{2 c^{* 2}}-i \frac{\epsilon \xi_{2}}{2 c^{* 2}}\right)
$$

The velocity of propagation is equal to $\left(c^{*}+\frac{\epsilon \xi_{1}}{2 c^{*}}\right)$ 
and the amplitude-attenuation factor is equal to

$\exp \left[\frac{\epsilon \chi \xi_{2} x}{2 c^{* 3}}\right]$ with $\xi_{2}<0$. The non-dimensional speed of propagation is computed for the following material parameters $\lambda=7.59 \times 10^{11} \mathrm{Dyn} \cdot \mathrm{cm}^{-2}$,

$\mu=1.89 \times 10^{11} \mathrm{Dyn} \cdot \mathrm{cm}^{-2}, \rho=2.7 \mathrm{~g} \cdot \mathrm{cm}^{-3}$,

$c_{E}=0.236 \mathrm{Cal} \cdot \mathrm{g}^{-1} \cdot \mathrm{C}^{-1}$.

$K^{*}=0.492 \mathrm{Cal} \cdot \mathrm{cm}^{-1} \cdot \mathrm{s}^{-1} \cdot{ }^{\circ} \mathrm{C}^{-1}, \quad \gamma=0.02, \quad T_{0}=20^{\circ} \mathrm{C}, x$ $=1 \mathrm{~cm}, c^{*}=0.9554$.

The non-dimensional speed of Rayleigh wave is shown graphically against the range $0.1 \leq \chi \leq 0.5$ of frequency in Figure 1, when two-temperature $a^{*}$ is 0.75 . With the increase in frequency, it increases very sharply at low frequency range and slowly for higher frequency range. The non-dimensional speed of Rayleigh wave is also shown graphically against the range

$0 \leq a^{*} \leq 1$ of two-temperature parameter in Figure 2, when the frequency $\chi=0.1$. With the increase in value of two-temperature parameter, it increases very slowly. It seems almost constant in Figure 2, but it increases for the whole range of the two-temperature parameter.

\section{Conclusion}

The appropriate solutions of the governing equations of two-temperature generalized thermoelastic medium without energy dissipation are applied at the boundary conditions at a thermally insulated free surface of a halfspace to obtain the frequency equation of Rayleigh wave. The frequency equation is approximated for the case of small thermal coupling and reduced for isotropic elastic case. From frequency equation of Rayleigh wave, it is observed that the phase speed of Rayleigh wave depends on various material parameters including the two-temperature parameter. The dependence of numerical values

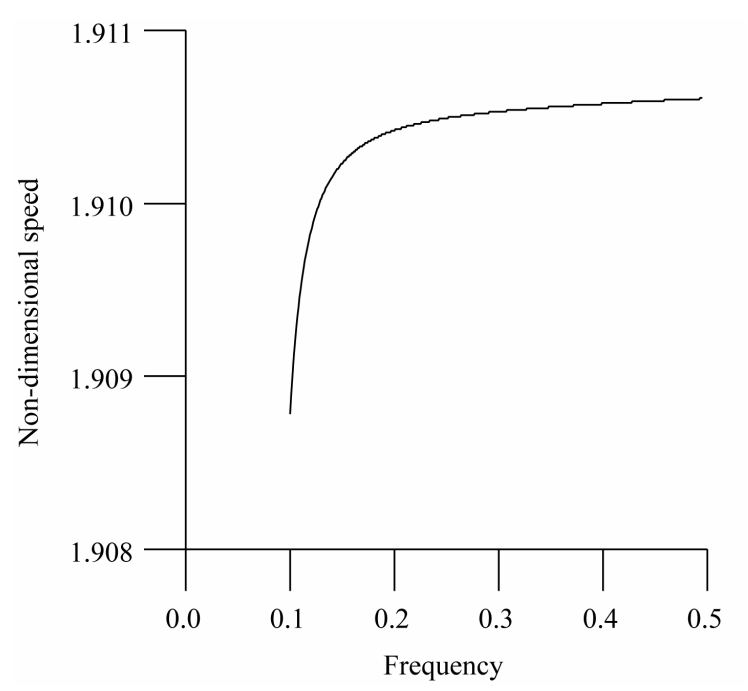

Figure 1. Variation of the non-dimensional speed of Rayleigh wave against frequency when $a^{*}=0.75$.

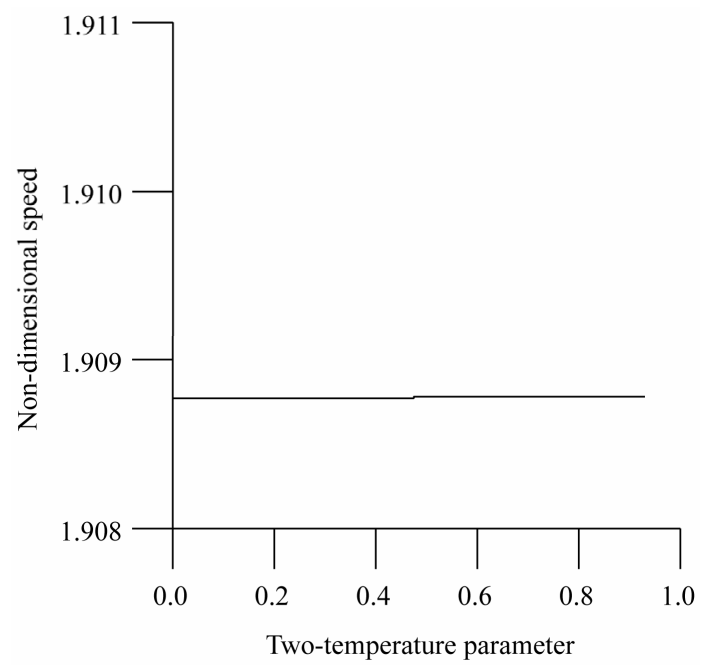

Figure 2. Variation of the non-dimensional speed of Rayleigh wave against two-temperature parameter when $\chi=$ 0.1.

of non-dimensional speed on the frequency and twotemperature parameter is shown graphically for a particular material representing the model.

\section{REFERENCES}

[1] H. Lord and Y. Shulman, "A Generalised Dynamical Theory of Thermoelasticity," Journal of the Mechanics and Physics of Solids, Vol. 15, No. 5, 1967, pp. 299-309. doi:10.1016/0022-5096(67)90024-5

[2] A. E. Green and K. A. Lindsay, "Thermoelasticity,” Journal of Elasticity, Vol. 2, No. 1, 1972, pp. 1-7. doi:10.1007/BF00045689

[3] J. Ignaczak and M. Ostoja-Starzewski, “Thermoelasticity with Finite Wave Speeds,” Oxford University Press, Oxford, 2009.

[4] A. E. Green and P. M. Naghdi, "Thermoelasticity without Energy Dissipation,” Journal of Elasticity, Vol. 31, No. 3, 1993, pp. 189-208. doi:10.1007/BF00044969

[5] R. B. Hetnarski and J. Ignaczak, "Generalized Thermoelasticity,” Journal of Thermal Stresses, Vol. 22, No. 4-5, 1999, pp. 451-476. doi:10.1080/014957399280832

[6] H. Deresiewicz, "Effect of Boundaries on Waves in a Thermo-Elastic Solid: Reflection of Plane Waves from Plane Boundary," Journal of the Mechanics and Physics of Solids, Vol. 8, No. 3, 1960, pp. 164-172. doi:10.1016/0022-5096(60)90035-1

[7] A. N. Sinha and S. B. Sinha, "Reflection of Thermoelastic Waves at a Solid Half Space with Thermal Relaxation," Journal of Physics of the Earth, Vol. 22, No. 2, 1974, pp. 237-244. doi:10.4294/jpe1952.22.237

[8] S. B. Sinha and K. A. Elsibai, "Reflection of Thermoelastic Waves at a Solid Half-Space with Two Thermal Relaxation Times,” Journal of Thermal Stresses, Vol. 19, No. 8, 1996, pp. 763-777. doi:10.1080/01495739608946205 
[9] S. B. Sinha and K. A. Elsibai, "Reflection and Refraction of Thermoelastic Waves at an Interface of Two Semi-Infinite Media with Two Thermal Relaxation Times," Journal of Thermal Stresses, Vol. 20, No. 2, 1997, pp. 129146. doi:10.1080/01495739708956095

[10] J. N. Sharma, V. Kumar and D. Chand, "Reflection of Generalized Thermoelastic Waves from the Boundary of a Half-Space," Journal of Thermal Stresses, Vol. 26, No. 10, 2003, pp. 925-942. doi:10.1080/01495730306342

[11] M. I. A. Othman and Y. Song, "Reflection of Plane Waves from an Elastic Solid Half-Space under Hydrostatic Initial Stress without Energy Dissipation,” International Journal of Solids and Structures, Vol. 44, No. 17, 2007, pp. 56515664. doi:10.1016/j.ijsolstr.2007.01.022

[12] B. Singh, "Effect of Hydrostatic Initial Stresses on Waves in a Thermoelastic Solid Half-Space," Applied Mathematics and Computation, Vol. 198, No. 2, 2008, pp. 494-505. doi:10.1016/j.amc.2007.08.072

[13] B. Singh, "Reflection of Plane Waves at the Free Surface of a Monoclinic Thermoelastic Solid Half-Space," European Journal of Mechanics-A/Solids, Vol. 29, No. 5, 2010, pp. 911-916. doi:10.1016/j.euromechsol.2010.05.005

[14] M. E. Gurtin and W. O. Williams, "On the Clausius-Duhem Inequality," Zeitschrift für Angewandte Mathematik und Physik ZAMP, Vol. 17, No. 5, 1966, pp. 626-633. doi:10.1007/BF01597243

[15] M. E. Gurtin and W. O. Williams, "An Axiomatic Foundation/or Continuum Thermodynamics," Archive for Rational Mechanics and Analysis, Vol. 26, No. 2, 1967, pp. 83-117. doi:10.1007/BF00285676

[16] P. J. Chen and M. E. Gurtin, "On a Theory of Heat Conduction Involving Two Temperatures," Zeitschrift für Angewandte Mathematik und Physik ZAMP, Vol. 19, No. 4, 1968, pp. 614-627. doi:10.1007/BF01594969

[17] P. J. Chen, M. E. Gurtin and W. O. Williams, "A Note on Non-Simple Heat Conduction," Zeitschrift für Angewandte Mathematik und Physik ZAMP, Vol. 19, No. 4, 1968, pp. 969-970. doi:10.1007/BF01602278

[18] P. J. Chen, M. E. Gurtin and W. O. Williams, "On the
Thermodynamics of Non-Simple Elastic Materials with Two-Temperatures," Zeitschrift für Angewandte Mathematik und Physik ZAMP, Vol. 20, No. 1, 1969, pp. 107112. doi:10.1007/BF01591120

[19] W. E. Warren and P. J. Chen, "Wave Propagation in the Two-Temperature Theory of Thermoelasticity," Acta Mechanica, Vol. 16, No. 1-2, 1973, pp. 21-33. doi:10.1007/BF01177123

[20] B. A. Boley and I. S. Tolins, "Transient Coupled Thermoplastic Boundary Value Problems in the Half-Space," Journal of Applied Mechanics, Vol. 29, No. 4, 1962, pp. 637-646. doi:10.1115/1.3640647

[21] P. Puri and P. M. Jordan, "On the Propagation of Harmonic Plane Waves under the Two-Temperature Theory," International Journal of Engineering Science, Vol. 44, No. 17, 2006, pp. 1113-1126. doi:10.1016/j.ijengsci.2006.07.002

[22] R. Quintanilla and P. M. Jordan, "A Note on the Two Temperature Theory with Dual-Phase-Lag Delay: Some Exact Solutions," Mechanics Research Communications, Vol. 36, No. 7, 2009, pp. 796-803. doi:10.1016/j.mechrescom.2009.05.002

[23] H. M. Youssef, "Theory of Two-Temperature Generalized Thermoelasticity," IMA Journal of Applied Mathematics, Vol. 71, No. 3, 2006, pp. 383-390. doi:10.1093/imamat/hxh101

[24] R. Kumar and S. Mukhopadhyay, "Effects of Thermal Relaxation Time on Plane Wave Propagation under TwoTemperature Thermoelasticity," International Journal of Engineering Science, Vol. 48, No. 2, 2010, pp. 128-139. doi:10.1016/j.ijengsci.2009.07.001

[25] A. Magãna and R. Quintanilla, "Uniqueness and Growth of Solutions in Two-Temperature Generalized Thermoelastic Theories," Mathematics and Mechanics of Solids, Vol. 14, No. 7, 2009, pp. 622-634. doi:10.1177/1081286507087653

[26] H. M. Youssef, “Theory of Two-Temperature Thermoelasticity without Energy Dissipation," Journal of Thermal Stresses, Vol. 34, No. 2, 2011, pp. 138-146. doi:10.1080/01495739.2010.511941 